Research Article

\title{
Investigation on the Mechanism of Qubi Formula in Treating Psoriasis Based on Network Pharmacology
}

\author{
Lin Zhou $\mathbb{D}^{1,}{ }^{1,2}$ Lingyun Zhang, ${ }^{3}$ and Disheng Tao $\mathbb{D}^{1,2}$ \\ ${ }^{1}$ Department of Dermatology and Venereal Diseases, Affiliated Hospital of Integrated Traditional Chinese and Western Medicine, \\ Nanjing University of Chinese Medicine, Nanjing 210028, China \\ ${ }^{2}$ Department of Dermatology and Venereal Diseases, Jiangsu Province Academy of Traditional Chinese Medicine, \\ Nanjing 210028, China \\ ${ }^{3}$ Nanjing Nuoyu Medical Service Co., Ltd., Jiangning Junhetang Traditional Chinese Medicine Clinic, Nanjing 210000, China \\ Correspondence should be addressed to Disheng Tao; tds2005800@126.com
}

Received 9 March 2020; Revised 18 May 2020; Accepted 23 May 2020; Published 22 June 2020

Guest Editor: Yuan Xu

Copyright (c) 2020 Lin Zhou et al. This is an open access article distributed under the Creative Commons Attribution License, which permits unrestricted use, distribution, and reproduction in any medium, provided the original work is properly cited.

Objective. To elucidate the pharmacological mechanisms of Qubi Formula (QBF), a traditional Chinese medicine (TCM) formula which has been demonstrated as an effective therapy for psoriasis in China. Methods. The Traditional Chinese Medicine Systems Pharmacology (TCMSP) database, BATMAN-TCM database, and literature search were used to excavate the pharmacologically active ingredients of QBF and to predict the potential targets. Psoriasis-related targets were obtained from Therapeutic Target Database (TTD), DrugBank database (DBD), MalaCards database, and DisGeNET database. Then, we established the network concerning the interactions of potential targets of QBF with well-known psoriasis-related targets by using protein-protein interaction (PPI) data in String database. Afterwards, topological parameters (including DNMC, Degree, Closeness, and Betweenness) were calculated to excavate the core targets of Qubi Formula in treating psoriasis (main targets in the PPI network). Cytoscape was used to construct the ingredients-targets core network for Qubi Formula in treating psoriasis, and ClueGO was used to perform GO-BP and KEGG pathway enrichment analysis on these core targets. Results. The ingredient-target-disease core network of QBF in treating psoriasis was screened to contain 175 active ingredients, which corresponded to 27 core targets. Additionally, enrichment analysis suggested that targets of QBF in treating psoriasis were mainly clustered into multiple biological processes (associated with nuclear translocation of proteins, cellular response to multiple stimuli (immunoinflammatory factors, oxidative stress, and nutrient substance), lymphocyte activation, regulation of cyclase activity, cell-cell adhesion, and cell death) and related pathways (VEGF, JAK-STAT, TLRs, NF- $\kappa \mathrm{B}$, and lymphocyte differentiation-related pathways), indicating the underlying mechanisms of QBF on psoriasis. Conclusion. In this work, we have successfully illuminated that Qubi Formula could relieve a wide variety of pathological factors (such as inflammatory infiltration and abnormal angiogenesis) of psoriasis in a "multicompound, multitarget, and multipathway" manner by using network pharmacology. Moreover, our present outcomes might shed light on the further clinical application of QBF on psoriasis treatment.

\section{Introduction}

Psoriasis is a common and frequently occurring disease in dermatology, which is characterized by easy diagnosis and difficult treatment, as well as recurrent disease course [1]. An epidemiological survey shows that the overall prevalence of psoriasis is approximately $0.5 \%$ in China [2]. The pathogenesis of psoriasis is still not completely clarified. Present studies have suggested that autoimmune disorders, dysfunction in various inflammatory signal transduction pathways, abnormal expression of psoriatic susceptibility gene, and obesity might be involved in the pathogenesis of psoriasis [3-9]. The unknown pathogenesis has brought difficulties to the treatment, without curative approaches against psoriasis at present. Traditional Chinese medicine (TCM) has unique advantages in treating psoriasis, which can play therapeutic roles through multiple targets and multiple pathways, corresponding to the dysfunction of various pathways 
underlying the pathogenesis of psoriasis [10-13]. However, TCM also has defects. Due to the unclear component entering the blood through the compound TCM, the mechanism of action is not completely clear, which restricts the further standardization and internationalization of TCM for psoriasis.

Qubi Formula (QBF) is an experience prescription for treating psoriasis at the Department of Dermatology in our hospital. It consists of Bubali Cornu (Shuiniujiao, SNJ, $30 \mathrm{~g}$ ), Rehmanniae Radix (Dihuang, DH, $20 \mathrm{~g}$ ), Paeoniae Radix Rubra (Chishao, CS, 10 g), Moutan Cortex (Mudanpi, MDP, 15 g), Arnebiae Radix (Zicao, ZC, 10 g), Lonicerae Japonicae Flos (Jinyinhua, JYH, $10 \mathrm{~g}$ ), Forsythiae Fructus (Lianqiao, LQ, $10 \mathrm{~g}$ ), Isatidis Radix (Banlangen, BLG, $30 \mathrm{~g}$ ), and Glycyrrhizae Radix et Rhizoma (Gancao, GC, 6g). QBF is modified from the classical TCM formula "Xi-Jiao-DiHuang decoction." In this formula, Bubali Cornu is used as the sovereign drug (Jun), while Rehmanniae Radix and Isatidis Radix are utilized as the minister herbs (Chen); Paeoniae Radix Rubra, Moutan Cortex, Arnebiae Radix, Lonicerae Japonicae Flos, and Forsythiae Fructus are the assistant herbs (Zuo), whereas Glycyrrhizae Radix et Rhizoma is the messenger herbs (Shi). The mixed application of these drugs exerts the effects of clearing away heat and removing toxicity, cooling blood, and receding speckles. The effects of QBF on psoriasis have been validated by clinical practice in multiple years, especially in patients with bloodheat subtypes of psoriasis. However, the scientific basis as well as potential pharmacological mechanisms of QBF is still unclear, which needs further investigations.

Conventional researches on the mechanism of one traditional Chinese medicine mostly follow the model of "one drug-one target-one disease," which cannot reflect the characteristics of TCM (multicompound, multitarget, and multipathway). Herein, in this study, a comprehensive approach [14] (a combination of multiple network-based computational and algorithm-based approaches) was utilized, by combining prediction of active compounds based on multiple pharmacokinetic parameters, excavation of diverse drug targets, and network analysis from a macroscopic perspective, aiming at the illumination of the underlying mechanisms of QBF on psoriasis and providing ideas for subsequent research.

\section{Materials and Methods}

2.1. Screening of Potential Pharmacological Active Ingredients and Targets of Qubi Formula. BATMAN-TCM [15] (http:// bionet.Ncpsb.Org/batman-tcm/index.Php/Home/Index/ index) is a bioinformatics analysis tool for analyzing pharmacological active ingredients of Chinese medicines. In order to obtain the information about the ingredients of QBF, "SHUI NIU JIAO, DI HUANG, CHI SHAO, MU DAN PI, ZI CAO, JIN YIN HUA, LIAN QIAO, BAN LAN GEN, and GAN CAO" were used as key words to search in the BATMAN database, giving rise to a total of 572 compounds.

The Traditional Chinese Medicine Systems Pharmacology (TCMSP) database [16] (http://tcmspw.com/tcmsp.php) is a unique platform which can provide pharmacokinetic properties (involving oral bioavailability, drug-likeness, aqueous solubility, etc.) and potential targets for natural compounds. We searched the above 572 compounds in the TCMSP platform to obtain their pharmacokinetic parameters. After obtaining the pharmacokinetic information on the 572 compounds, the reference was screened according to oral bioavailability $(\mathrm{OB}) \geq 30 \%$ and drug-likeness $(\mathrm{DL}) \geq 0.18$ (mean value for all molecules within the DrugBank database), aiming to screen potential pharmacologically active ingredients of QBF [17]. In this study, these cut-off values utilized helped to efficiently and maximally collect data from QBF using the least components, and the pharmacokinetic data reported may account for this [18]. OB [19], defined as the distribution degree of an oral dose of drug into bloodstream, is one of the most requisite premises in terms of oral drug discovery as well as clinical application. Additionally, drug-likeness, which is defined as a qualitative concept for assessment of the structural similarity of compounds with clinical therapeutics in the DrugBank database, is determined early after drug discovery [20]. In addition, through literature review, certain compounds with the $\mathrm{OB}<30$ or $\mathrm{DL}<0.18$ but with extensive pharmaceutical activities (such as oleic acid [21] and arnebinol [22]), or those with relatively higher contents (like jioglutin and alkannin) or those used for the quality identification of single herb in the Pharmacopoeia (goitrin), were also added as potential pharmacologically active ingredients of QBF.

Apart from assisting in exploring the active ingredients of TCM, TCMSP databases could also predict the potential targets of compounds based on SysDT model, HIT database, reverse molecular docking, etc. [23].

\subsection{Collection of Known Psoriasis-Related Targets, Psoriasis.} In order to obtain the known psoriasis-related targets, "psoriasis" was used as key word to search in Therapeutic Target Database (TTD) [24], DrugBank database [25], MalaCards database [26], and DisGeNET database [27]. After searching in the DisGeNET database, results were sorted by the disease specificity index (DSI), following by the removal of targets lower than the median of DSI obtained from all the known psoriasis-related genes. Additionally, the drugs with abnormal status in TTD and DrugBank database and their corresponding targets were also taken out. The detailed information of these known psoriasis-related was summarized in Table S1 after redundancy deletion.

\subsection{Excavation of Core Targets of QBF for Treating Psoriasis} and Construction of Core Network on Active IngredientsTargets. First, targets obtained from the above two steps (potential targets of QBF and known psoriasis-related targets) were standardized in the UniProt database [28] by selecting the species "Homo sapiens," aiming to acquire the single universal gene names. Then, both potential targets of QBF and known psoriasis-related targets were uploaded to the online Wayne diagram tool (http://bioinfogp.cnb.csic.es/ tools/venny/index.html, Version 2.1.0) for mapping; that is, targets from these two sets were intersected to obtain the candidate targets of QBF for treating psoriasis. 
Subsequently, the candidate targets were imported into the String database [29] to obtain protein-protein interactions (PPIs) by setting the minimum value of the combined score at 0.400 and the species as "Homo sapiens." The topological parameters (DMNC, Degree, Closeness, and Betweenness) of each target (node) in the network were calculated using the cytoHubba plugin [30]. The median values of these four parameters of all nodes were used as a screening condition. Nodes with all the four parameter greater than the median values were considered as the main hubs that played core roles in the PPI network, that is, the core targets of QBF for psoriasis. Finally, Cytoscape was used to construct the core network of active ingredients-targets.

2.4. Enrichment Analysis of Core Targets. The ClueGO [31] plugin from Cytoscape software, integrative GO-biological process (BP), and KEGG database were applied to perform enrichment analysis on the core targets, and species was selected as "Homo" in the ClueGO interface. All the core targets were sequentially imported, followed by enrichment analysis. $\kappa$ value was defaulted at 0.4 and $p$ was set at $\leq 0.05$, which were used as the screening conditions for plotting enrichment analysis.

\section{Results}

3.1. Screening of Potential Pharmacologically Active Ingredients and Their Targets of Qubi Formula. The search through the BATMAN-TCM database revealed a total of 572 compounds of QBF. Accumulative efforts have been made to clarify the therapeutic mechanisms of TCM, however, with sluggish progress on the molecular level. Due to the unavailable effective methods specifically developed for the identification of the active compounds in medicinal herbs, OB screening combined with drug-likeness assessment may be a feasible strategy. In this study, $O B \geq 30 \%$ and $\mathrm{DL} \geq 0.18$ were used as the screening conditions. Then, a total of 202 possible compounds with proper values of above two parameters were collected for potential pharmacologically active ingredients from the herbal constituents of QBF. In addition, among the compounds that were screened out, we found another 35 compounds through the literature research of PubMed. Although these 35 compounds did not meet the screening conditions of $\mathrm{OB}$ and $\mathrm{DL}$, they were reported to use a wide range of pharmacological activities and were thus included in potential pharmacologically active ingredients. Finally, the active ingredients of SNJ, GC, CS, DH, MDP, ZC, JYH, LQ, and BLG were $6,97,26,6,12,17,30,27$, and 51, respectively. The proportions of the active ingredients in the sovereign drug (Jun), minister herbs (Chen), assistant herbs (Zuo), and messenger herbs (Shi) in all the 175 ingredients were $3.4 \%, 30.86 \%, 53.14 \%$, and $55.43 \%$, respectively. Among them, some compounds were widely present in multiple herbs of QBF, such as oleic acid, methyl linolenate, sitosterol, and paeoniflorin. The basic information of the potential pharmacologically active ingredients of Qubi Formula is shown in Table 1.
Subsequently, we explored the potential targets of the 237 potential pharmacologically active ingredients by excavating TCMSP databases, which yielded to 939 targets (shown in Table S2). The numbers of potential targets linked by SNJ, GC, CS, DH, MDP, ZC, JYH, LQ, and BLG were 668, $234,148,49,207,90,219,229$, and 111, respectively. The proportions of the potential targets of the sovereign drug (Jun), minister herbs (Chen), assistant herbs (Zuo), and messenger herbs (Shi) in all the 939 targets were $71.14 \%$, $11.93 \%, 30.35 \%$, and $24.92 \%$, respectively. Although the number of targets correlated with each herb of QBF is different, significant overlaps were observed in the nine herbs, which was suggestive of the congenerous or antergic roles of the various components in QBF via the regulation of similar targets.

In order to holistically and systemically obtain comprehensive understanding of the ingredient-target network in QBF, a network map was constructed by using Cytoscape, including 6273 edges and 1212 nodes (Figure 1). To be specific, the node degree indicated the number of target or edge correlated with the node according to topological analysis. A total of 142 ingredients were found in the as-established network to have the median of $\geq 18$ degrees. Of them, quercetin, arginine, and oleic acids kaempferol and luteolin acted on 166, 96, and 54 targets, respectively, which were subsequently considered as the critical pharmacologically active ingredients of QBF.

\subsection{Excavation of the Core Targets of Qubi Formula in Treating} Psoriasis. Psoriasis has been recognized as the polygenic disorder. In addition, the investigation of the interactions between genes as well as gene and environment could be used to reveal the pathogenesis of psoriasis. After the targets with abnormal status from TTD and DrugBank database and DSI $<0.535$ (the median of DSI) from DisGeNET database were excluded, we collected 605 targets (Table S1) associated with psoriasis from the four accessible resources. Notably, 104 of the identified potential targets of the QBF were also the well-recognized psoriasis disease- (or therapeutic drugs) related targets (Table S3 and Figure 2(a)). And these 104 targets were defined as the candidate targets for QBF in treating psoriasis.

Subsequently, to further select the core targets of QBF in treating psoriasis, the String database was used to construct the PPI network of the above targets (Figure 2(b)), followed by the calculation of the topological parameters (DMNC, Degree, Closeness, and Betweenness) of each node in the network using the cytoHubba plugin. The median values of these four parameters of all nodes were used as a screening condition. Nodes with all the four parameters greater than the median values were considered as the main hubs that played core roles in the PPI network. As a result, 27 targets (Table 2 and Table S4) were screened from the 104 candidate targets based on the values of topological parameters (Figure 2(c)), that is the core targets of QBF in treating psoriasis. 
TABLE 1: All the potential pharmacologically active ingredients of QBF.

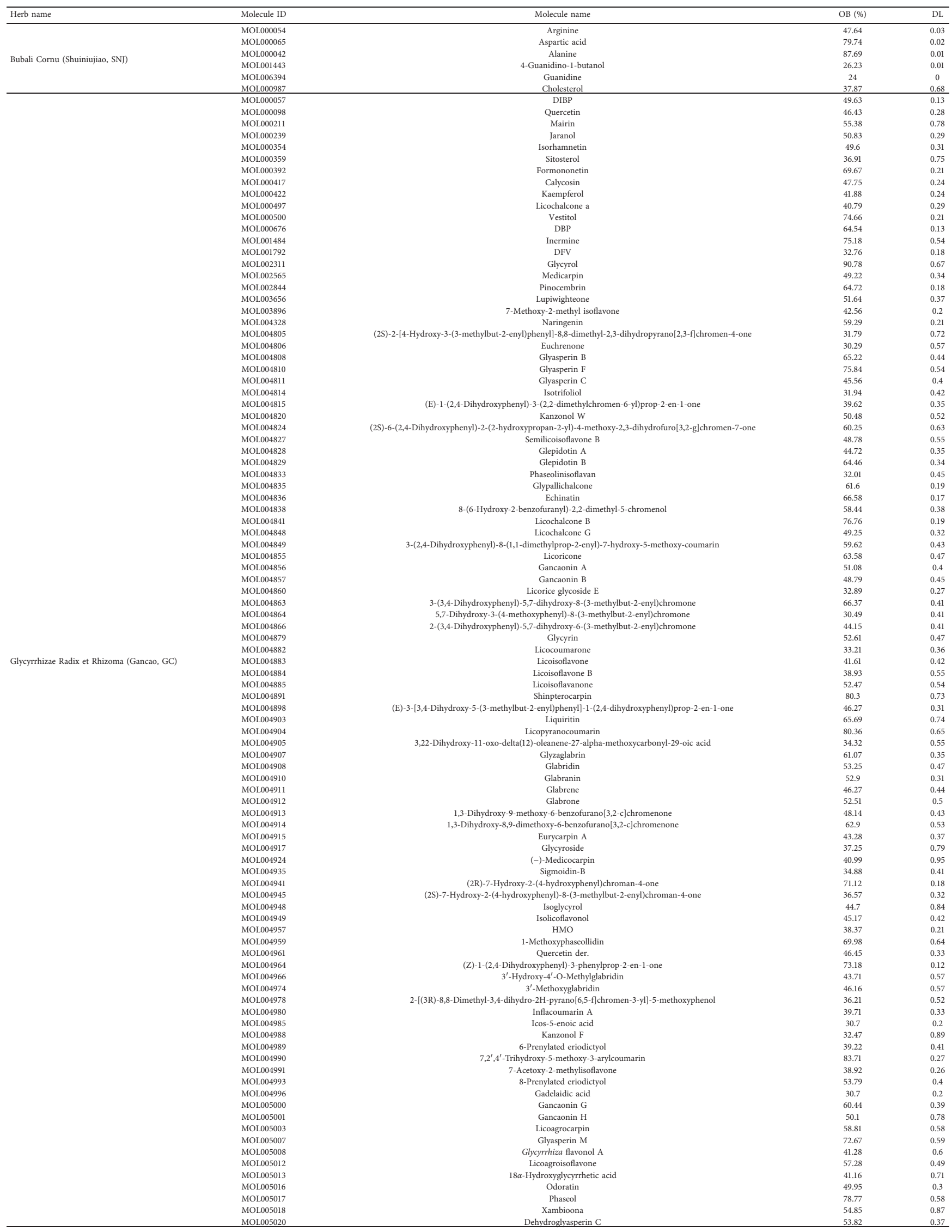


TABLE 1: Continued.

\begin{tabular}{|c|c|c|c|c|}
\hline Herb name & Molecule ID & Molecule name & $\mathrm{OB}(\%)$ & DL \\
\hline \multirow{26}{*}{ Paeoniae Radix Rubra (Chishao, CS) } & MOL001924 & Paeoniflorin & 53.87 & 0.79 \\
\hline & MOL000449 & Stigmasterol & 43.83 & 0.76 \\
\hline & MOL004355 & Spinasterol & 42.98 & 0.76 \\
\hline & MOL000358 & Beta-sitosterol & 36.91 & 0.75 \\
\hline & MOL000359 & Sitosterol & 36.91 & 0.75 \\
\hline & MOL002776 & Baicalin & 40.12 & 0.75 \\
\hline & MOL006999 & Stigmast-7-en-3-ol & 37.42 & 0.75 \\
\hline & MOL005043 & Campest-5-en-3beta-ol & 37.58 & 0.71 \\
\hline & MOL007003 & Benzoyl paeoniflorin & 31.14 & 0.54 \\
\hline & MOL007025 & Isobenzoyl paeoniflorin & 31.14 & 0.54 \\
\hline & MOL001002 & Ellagic acid & 43.06 & 0.43 \\
\hline & MOL001918 & Paeoniflorgenone & 87.59 & 0.37 \\
\hline & MOL007016 & Paeoniflorigenone & 65.33 & 0.37 \\
\hline & MOL006996 & 1-o-Beta-d-glucopyranosylpaeonisuffrone_qt & 65.08 & 0.35 \\
\hline & MOL007005 & Albiflorin_qt & 48.7 & 0.33 \\
\hline & MOL006992 & (2R,3 R)-4-Methoxyl-distylin & 59.98 & 0.3 \\
\hline & MOL006994 & 1-o-Beta-d-glucopyranosyl-8-o-benzoylpaeonisuffrone_qt & 36.01 & 0.3 \\
\hline & MOL007018 & 9-Ethyl-neo-paeoniaflorin A_qt & 64.42 & 0.3 \\
\hline & MOL006990 & $(1 \mathrm{~S}, 2 \mathrm{~S}, 4 \mathrm{R})$-trans-2-hydroxy-1,8-cineole-B-D-glucopyranoside & 30.25 & 0.27 \\
\hline & MOL000492 & $(+)$-catechin & 54.83 & 0.24 \\
\hline & MOL002714 & Baicalein & 33.52 & 0.21 \\
\hline & MOL002883 & Ethyl oleate (NF) & 32.4 & 0.19 \\
\hline & MOL001641 & Methyl linoleate & 41.93 & 0.17 \\
\hline & MOL000131 & EIC & 41.9 & 0.14 \\
\hline & MOL000675 & Oleic acid & 33.13 & 0.14 \\
\hline & MOL001746 & ELD & 31.2 & 0.14 \\
\hline & MOL000449 & Stigmasterol & 43.83 & 0.76 \\
\hline & MOL000359 & Sitosterol & 36.91 & 0.75 \\
\hline & MOL000131 & EIC & 41.9 & 0.14 \\
\hline Rehmanniae Radix (Dihuang, DH) & MOL003708 & Jioglutin D & 39.02 & 0.14 \\
\hline & MOL003689 & Aeginetic acid & 48.31 & 0.13 \\
\hline & MOL003706 & Jioglutin B & 90.71 & 0.13 \\
\hline & MOL000211 & Mairin & 55.38 & 0.78 \\
\hline & MOL000359 & Sitosterol & 36.91 & 0.75 \\
\hline & MOL007003 & Benzoyl paeoniflorin & 31.14 & 0.54 \\
\hline & MOL007369 & 4-O-methylpaeoniflorin_qt & 67.24 & 0.43 \\
\hline & MOL001925 & Paeoniflorin_qt & 68.18 & 0.4 \\
\hline & MOL007382 & Mudanpioside-h_qt 2 & 42.36 & 0.37 \\
\hline Moutan Cortex (Mudanpi, MDP) & MOL007384 & Paeonidanin_qt & 65.31 & 0.35 \\
\hline & MOL007374 & 5-[[5-(4-Methoxyphenyl)-2-furyl] methylene]barbituric acid & 43.44 & 0.3 \\
\hline & MOL000098 & Quercetin & 46.43 & 0.28 \\
\hline & MOL000422 & Kaempferol & 41.88 & 0.24 \\
\hline & MOL000492 & (+)-Catechin & 54.83 & 0.24 \\
\hline & MOL000675 & Oleic acid & 33.13 & 0.14 \\
\hline & MOL000359 & Sitosterol & 36.91 & 0.75 \\
\hline & MOL002372 & $(6 \mathrm{Z}, 10 \mathrm{E}, 14 \mathrm{E}, 18 \mathrm{E})-2,6,10,15,19,23$-Hexamethyltetracosa-2,6,10,14,18,22-hexaene & 33.55 & 0.42 \\
\hline & MOL007736 & Lithospermidin B & 60.48 & 0.39 \\
\hline & MOL007728 & Lithospermidin A & 75.08 & 0.38 \\
\hline & MOL007714 & 1-Methoxyacetylshikonin & 73.09 & 0.29 \\
\hline & MOL007715 & [(1R)-1-(5,8-Dihydroxy-1,4-dioxo-2-naphthyl)-4-methyl-pent-3-enyl] propanoate & 54.64 & 0.29 \\
\hline & MOL007716 & Acetylshikonin & 62.39 & 0.27 \\
\hline & MOL007734 & 5-[(E)-5-3-Furyl)-2-methyl-pent-2-enyl]-2,3-dimethoxy-p-benzoquinone & 61.8 & 0.24 \\
\hline Arnebiae Radix (Zicao, ZC) & MOL007722 & Isoarnebin 4 & 64.79 & 0.2 \\
\hline & MOL007735 & Des-O-methyllasiodiplodin & 30.12 & 0.2 \\
\hline & MOL001494 & Mandenol & 42 & 0.19 \\
\hline & MOL002883 & Ethyl oleate (NF) & 32.4 & 0.19 \\
\hline & MOL007719 & Arnebin 7 & 73.85 & 0.18 \\
\hline & MOL007717 & Alkannin & 6.09 & 0.35 \\
\hline & MOL000131 & EIC & 41.9 & 0.14 \\
\hline & MOL000675 & Oleic acid & 33.13 & 0.14 \\
\hline & MOL007731 & Arnebinol & 56.66 & 0.14 \\
\hline & MOL003036 & $\begin{array}{r}\text { (3S,8 R,9R,10 R,13R,14S,17R)-17-[(E,2R,5S)-5-Ethyl-6-methylhept-3-en-2-yl]-10,13-dimethyl-2,3,4,7,8,9,11,12,14,15,16, } \\
\text { 17-dodecahydro-1H-cyclopenta[a]phenanthren-3-ol }\end{array}$ & 43.83 & 0.76 \\
\hline & MOL000449 & Stigmasterol & 43.83 & 0.76 \\
\hline & MOL000358 & Beta-sitosterol & 36.91 & 0.75 \\
\hline & MOL003108 & Caeruloside C & 55.64 & 0.73 \\
\hline & MOL003124 & Xylostosidine & 43.17 & 0.64 \\
\hline & MOL002773 & Beta-carotene & 37.18 & 0.58 \\
\hline & MOL003101 & 7-Epivogeloside & 46.13 & 0.58 \\
\hline & MOL003059 & Kryptoxanthin & 47.25 & 0.57 \\
\hline & MOL003062 & $4,5^{\prime}$-Retro-beta.,,beta-carotene-3,3'-dione, $4^{\prime}, 5^{\prime}$-didehydro- & 31.22 & 0.55 \\
\hline & MOL002707 & Phytofluene & 43.18 & 0.5 \\
\hline & MOL003111 & Centauroside_qt & 55.79 & 0.5 \\
\hline & MOL003128 & Dinethylsecologanoside & 48.46 & 0.48 \\
\hline & MOL003095 & 5-Hydroxy-7-methoxy-2-(3,4,5-trimethoxyphenyl)chromone & 51.96 & 0.41 \\
\hline & MOL003014 & Secologanic dibutylacetal_qt & 53.65 & 0.29 \\
\hline & MOL000098 & Quercetin & 46.43 & 0.28 \\
\hline Lonicerae Japonicae Flos (Jinyinhua, JYH) & MOL003044 & Chrysoeriol & 35.85 & 0.27 \\
\hline & MOL000006 & Luteolin & 36.16 & 0.25 \\
\hline & MOL002914 & Eriodyctiol (flavanone) & 41.35 & 0.24 \\
\hline & MOL000422 & Kaempferol & 41.88 & 0.24 \\
\hline & MOL003006 & $\begin{array}{l}\text { (-)-(3R,8S,9R,9aS,10aS)-9-Ethenyl-8-(beta-D-glucopyranosyloxy)-2,3,9,9a,10, } \\
\text { 10a-hexahydro-5-oxo-5H,8H-pyrano[4,-dloxazolo[3,2-al pyridine-3-carboxylic acid_at }\end{array}$ & 87.47 & 0.23 \\
\hline & MOL001495 & $\begin{array}{l}\text { 10a-hexahydro-5-oxo-5H,8H-pyrano[4,3-d]oxazolo[3,2-a]pyridine-3-carboxylic acid_qt } \\
\text { Ethyl linolenate }\end{array}$ & 46.1 & 0.2 \\
\hline & MOL001494 & Mandenol & 42 & 0.19 \\
\hline & MOL003117 & Ioniceracetalide B_qt & 61.19 & 0.19 \\
\hline & MOL001398 & Methyl linolenate & 46.15 & 0.17 \\
\hline & MOL001641 & Methyl linoleate & 41.93 & 0.17 \\
\hline & MOL003103 & Methyl octadeca-8,11-dienoate & 41.93 & 0.17 \\
\hline & MOL003120 & Loniceracetalide A_qt & 89.38 & 0.17 \\
\hline & MOL002003 & (-)-Caryophyllene oxide & 32.67 & 0.13 \\
\hline & MOL000266 & Beta-cubebene & 32.81 & 0.11 \\
\hline & MOL002697 & Junipene & 44.07 & 0.11 \\
\hline
\end{tabular}


TABle 1: Continued.

\begin{tabular}{|c|c|c|c|c|}
\hline Herb name & Molecule ID & Molecule name & $\mathrm{OB}(\%)$ & DL \\
\hline \multirow{27}{*}{ Forsythiae Fructus (Lianqiao, LQ) } & MOL000791 & Bicuculline & 69.67 & 0.88 \\
\hline & MOL003305 & Phillyrin & 36.4 & 0.86 \\
\hline & MOL003365 & Lactucasterol & 40.99 & 0.85 \\
\hline & MOL000522 & Arctiin & 34.45 & 0.84 \\
\hline & MOL003281 & 20(S)-Dammar-24-ene-3 $\beta, 20$-diol-3-acetate & 40.23 & 0.82 \\
\hline & MOL003315 & 3beta-Acetyl-20,25-epoxydammarane-24alpha-ol & 33.07 & 0.79 \\
\hline & MOL000211 & Mairin & 55.38 & 0.78 \\
\hline & MOL000358 & Beta-sitosterol & 36.91 & 0.75 \\
\hline & MOL003344 & $\beta$-Amyrin acetate & 42.06 & 0.74 \\
\hline & MOL003348 & Adhyperforin & 44.03 & 0.61 \\
\hline & MOL003347 & Hyperforin & 44.03 & 0.6 \\
\hline & MOL003295 & (+)-Pinoresinol monomethyl ether & 53.08 & 0.57 \\
\hline & MOL003306 & ACon1_001697 & 85.12 & 0.57 \\
\hline & MOL003308 & (+)-Pinoresinol monomethyl ether-4-D-beta-glucoside_qt & 61.2 & 0.57 \\
\hline & MOL003322 & Forsythinol & 81.25 & 0.57 \\
\hline & MOL003330 & $(-)$-Phillygenin & 95.04 & 0.57 \\
\hline & MOL003290 & (3R,4 R)-3,4-bis[(3,4-Dimethoxyphenyl)methyl] oxolan-2-one & 52.3 & 0.48 \\
\hline & MOL003283 & $(2 \mathrm{R}, 3 \mathrm{R}, 4 \mathrm{~S})-4$-(4-Hydroxy-3-methoxy-phenyl)-7-methoxy-2,3-dimethylol-tetralin-6-ol & 66.51 & 0.39 \\
\hline & MOL003370 & Onjixanthone I & 79.16 & 0.3 \\
\hline & MOL000098 & Quercetin & 46.43 & 0.28 \\
\hline & MOL000006 & Luteolin & 36.16 & 0.25 \\
\hline & MOL000422 & Kaempferol & 41.88 & 0.24 \\
\hline & MOL000173 & Wogonin & 30.68 & 0.23 \\
\hline & MOL003358 & Euxanthone & 92.98 & 0.16 \\
\hline & MOL003302 & Forsythidmethylester_qt & 121.84 & 0.12 \\
\hline & MOL003360 & Norlapachol & 46.99 & 0.11 \\
\hline & MOL003300 & Forsythide_qt & 46.6 & 0.1 \\
\hline \multirow{51}{*}{ Isatidis Radix (Banlangen, BLG) } & MOL001810 & 6-(3-Oxoindolin-2-ylidene)indolo[2,1-b]quinazolin-12-one & 45.28 & 0.89 \\
\hline & MOL001806 & Stigmasta-5,22-diene-3beta,7beta-diol & 42.56 & 0.83 \\
\hline & MOL001804 & Stigmasta-5,22-diene-3beta,7alpha-diol & 43.04 & 0.82 \\
\hline & MOL001755 & 24-Ethylcholest-4-en-3-one & 36.08 & 0.76 \\
\hline & MOL000449 & Stigmasterol & 43.83 & 0.76 \\
\hline & MOL001771 & Poriferast-5-en-3beta-ol & 36.91 & 0.75 \\
\hline & MOL001800 & Rosasterol & 35.87 & 0.75 \\
\hline & MOL000358 & Beta-sitosterol & 36.91 & 0.75 \\
\hline & MOL000359 & Sitosterol & 36.91 & 0.75 \\
\hline & MOL002322 & Isovitexin & 31.29 & 0.72 \\
\hline & MOL001790 & Linarin & 39.84 & 0.71 \\
\hline & MOL000953 & CLR & 37.87 & 0.68 \\
\hline & MOL001769 & Beta-sitosterol decantate & 34.57 & 0.57 \\
\hline & MOL001783 & 2-(9-((3-Methyl-2-oxopent-3-en-1-yl)oxy)-2-oxo-1,2,8,9-tetrahydrofuro[2,3-h] quinolin-8-yl)propan-2-yl acetate & 64 & 0.57 \\
\hline & MOL001828 & 3-[(3,5-Dimethoxy-4-oxo-1-cyclohexa-2,5-dienylidene)methyl]-2,4-dihydro-1H-pyrrolo[2,1-b]quinazolin-9-one & 51.84 & 0.56 \\
\hline & MOL001811 & Goitrin & 3.23 & 0.01 \\
\hline & MOL001750 & Glucobrassicin & 66.02 & 0.48 \\
\hline & MOL001734 & 3-[[(2R,3R,5R,6S)-3,5-Dihydroxy-6-(1H-indol-3-yloxy)-4-oxooxan-2-yl]methoxy]-3-oxopropanoic acid & 85.87 & 0.47 \\
\hline & MOL001779 & Sinoacutine & 49.11 & 0.46 \\
\hline & MOL001803 & Sinensetin & 50.56 & 0.45 \\
\hline & MOL001721 & Isaindigodione & 60.12 & 0.41 \\
\hline & MOL001733 & Eupatorin & 30.23 & 0.37 \\
\hline & MOL001749 & ZINC03860434 & 43.59 & 0.35 \\
\hline & MOL001793 & (E)-2-[(3-Indole) cyanomethylene- $]$-3-indolinone & 54.59 & 0.32 \\
\hline & MOL001722 & 2-O-beta-D-Glucopyranosyl-2H-1,4-benzoxazin-3(4H)-one & 43.62 & 0.31 \\
\hline & MOL001767 & Hydroxyindirubin & 63.37 & 0.3 \\
\hline & MOL001774 & Ineketone & 37.14 & 0.3 \\
\hline & MOL001735 & Dinatin & 30.97 & 0.27 \\
\hline & MOL001736 & (-)-Taxifolin & 60.51 & 0.27 \\
\hline & MOL001798 & Neohesperidin_qt & 71.17 & 0.27 \\
\hline & MOL001781 & Indigo & 38.2 & 0.26 \\
\hline & MOL001782 & (2Z)-2-(2-Oxoindolin-3-ylidene)indolin-3-one & 48.4 & 0.26 \\
\hline & MOL001814 & (E)-3-(3,5-Dimethoxy-4-hydroxy-benzylidene)-2-indolinone & 57.18 & 0.25 \\
\hline & MOL001820 & (E)-3-(3,5-Dimethoxy-4-hydroxyb-enzylidene)-2-indolinone & 65.17 & 0.25 \\
\hline & MOL001689 & Acacetin & 34.97 & 0.24 \\
\hline & MOL001833 & Glucobrassicin-1-sulfonate_qt & 42.52 & 0.24 \\
\hline & MOL001756 & Quindoline & 33.17 & 0.22 \\
\hline & MOL001728 & 3-[ $2^{\prime}-\left(5^{\prime}\right.$-Hydroxymethyl)furyl]-1(2H)-isoquinolinone-7-O-beta-D-glucoside_qt & 51.74 & 0.18 \\
\hline & MOL001792 & $\mathrm{DFV}$ & 32.76 & 0.18 \\
\hline & MOL001398 & Methyl linolenate & 46.15 & 0.17 \\
\hline & MOL001745 & Methyl vaccenate & 31.9 & 0.17 \\
\hline & MOL001748 & Methyl (E)-octadec-8-enoate & 31.9 & 0.17 \\
\hline & MOL001763 & 3-(2-Hydroxyphenyl)quinazolin-4-one & 63.58 & 0.16 \\
\hline & MOL001789 & Isoliquiritigenin & 85.32 & 0.15 \\
\hline & MOL001821 & Methyl 2-ethylhexyl phthalate & 65.98 & 0.15 \\
\hline & MOL000432 & Linolenic acid & 45.01 & 0.15 \\
\hline & MOL000131 & EIC & 41.9 & 0.14 \\
\hline & MOL001746 & ELD & 31.2 & 0.14 \\
\hline & MOL000057 & DIBP & 49.63 & 0.13 \\
\hline & MOL000676 & DBP & 64.54 & 0.13 \\
\hline & MOL001818 & Methyl palmitelaidate & 34.61 & 0.12 \\
\hline
\end{tabular}

3.3. Construction of Active Ingredients-Targets Core Network for Qubi Formula in Treating Psoriasis. In order to further understand the "multicompound and multitarget" mechanism of Qubi Formula in treating psoriasis, we searched for the potential ingredients of Qubi Formula which could affect the 27 core targets based on the relationship between the ingredients and their targets, followed by construction of the core network on active ingredients-targets (Figure 3(a)) using Cytoscape software and the statistical analysis of the degree of each node in the network. As shown in Figure 3(b), the degrees of active ingredients ranged from 1 to 18 in the core network, with the median of 2 , indicating that more than half of the compounds acted on more than one target, while the degree of the target ranged from 1 to 160 , with the median of 2. Among all the active ingredients, the top 4 were quercetin, luteolin, kaempferol, and wogonin in terms of Degree. Previous preclinical studies have confirmed that all of them could delay the progression of psoriasis and relieve symptoms in multiple animal models [32-34]. Among all the targets, the top three were PTGS2, MAPK14, and NOS3 in terms of Degree, and all of them have been demonstrated to play important roles in the pathogenesis of psoriasis, such as inflammatory infiltration, abnormal differentiation of keratinocytes, and oxidative stress injury $[35,36]$. 


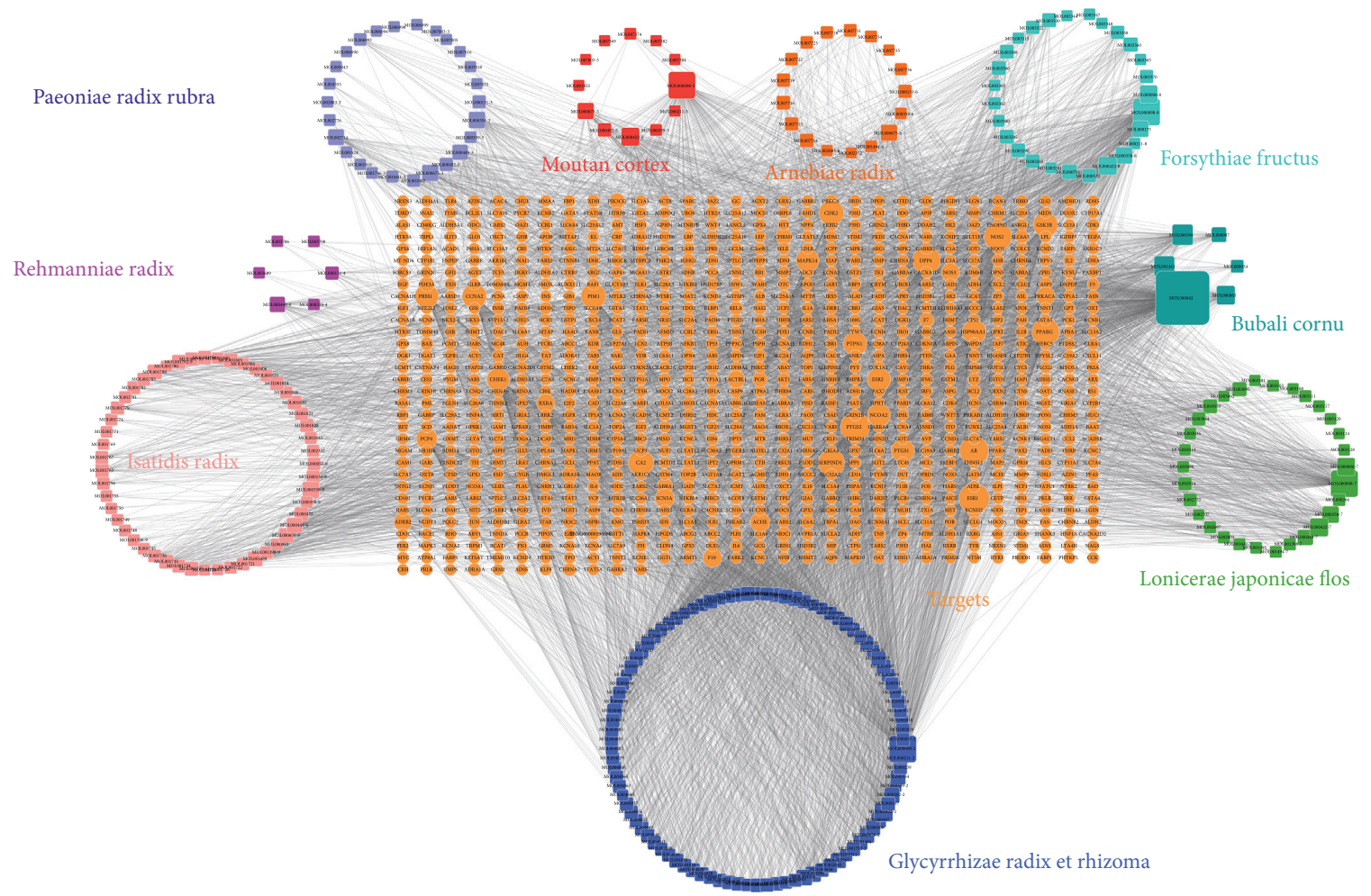

FIGURE 1: Construction of the QBF compound-potential target network. The compound-potential target network was constructed by linking the candidate compounds and their potential targets of the 9 herbs, which are constituents of QBF. The nodes representing candidate compounds are shown as polychrome square and the targets are indicated by orange circle.
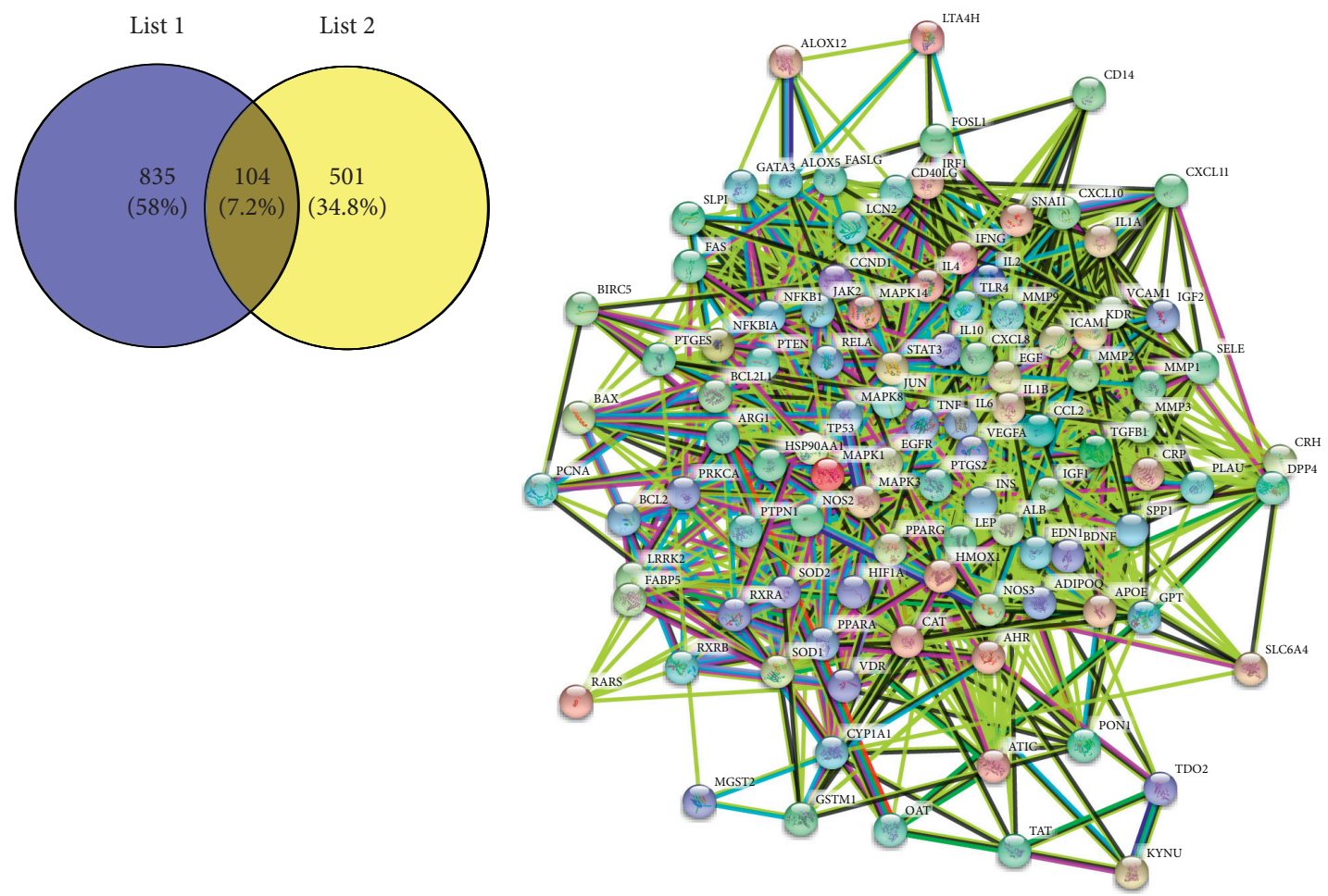

(a)

(b)

FIGURE 2: Continued. 


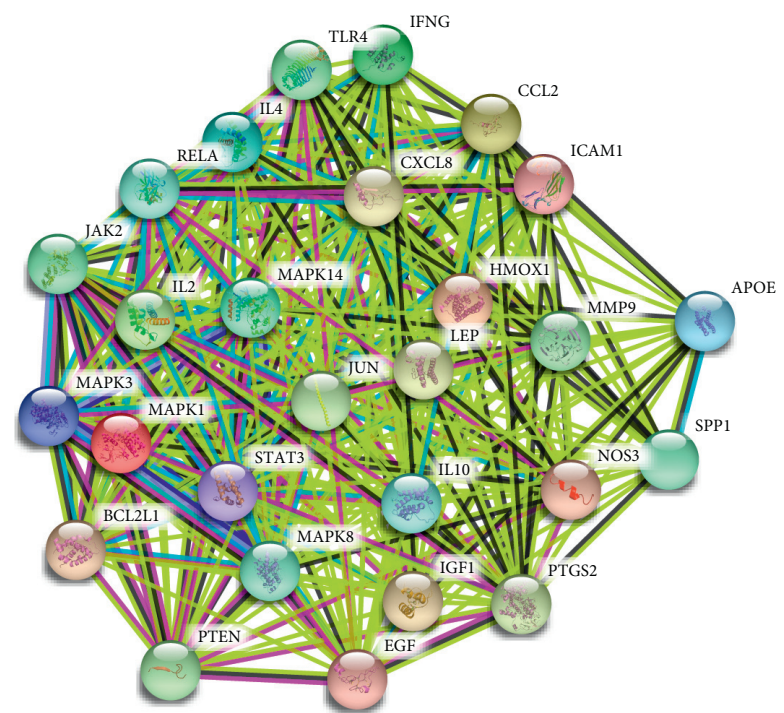

(c)

FIgURE 2: Excavation of the core target of QBF in treating psoriasis. (a) The Venn diagram showed that QBF shared 104 potential targets with known pathological course-related targets of psoriasis. (b) The PPI network of all the candidate targets of QBF in treating psoriasis. (c) The PPI of the core target of QBF in treating psoriasis.

TABLE 2: Topological feature values of all the core targets for QBF against psoriasis.

\begin{tabular}{lcccc}
\hline Node name & DMNC & Degree & Closeness & Betweenness \\
\hline APOE & 1.19 & 47.00 & 74.33 & 41.76 \\
BCL2L1 & 1.19 & 49.00 & 75.33 & 46.09 \\
CCL2 & 1.15 & 73.00 & 87.50 & 105.15 \\
CXCL8 & 1.12 & 75.00 & 88.50 & 144.89 \\
EGF & 1.18 & 63.00 & 82.33 & 83.27 \\
HMOX1 & 1.19 & 56.00 & 79.00 & 64.03 \\
ICAM1 & 1.24 & 64.00 & 82.83 & 48.67 \\
IFNG & 1.21 & 62.00 & 81.83 & 59.30 \\
IGF1 & 1.23 & 62.00 & 81.83 & 48.60 \\
IL10 & 1.13 & 74.00 & 88.00 & 128.25 \\
IL2 & 1.17 & 62.00 & 81.83 & 117.83 \\
IL4 & 1.20 & 65.00 & 83.33 & 67.65 \\
JAK2 & 1.24 & 48.00 & 74.67 & 47.23 \\
JUN & 1.15 & 74.00 & 88.00 & 111.79 \\
LEP & 1.17 & 59.00 & 80.50 & 68.01 \\
MAPK1 & 1.14 & 67.00 & 84.33 & 135.31 \\
MAPK14 & 1.23 & 56.00 & 78.83 & 41.89 \\
MAPK3 & 1.12 & 73.00 & 87.33 & 164.81 \\
MAPK8 & 1.14 & 71.00 & 86.50 & 114.31 \\
MMP9 & 1.13 & 73.00 & 87.50 & 225.47 \\
NOS3 & 1.21 & 55.00 & 78.33 & 50.16 \\
PTEN & 1.17 & 49.00 & 75.33 & 46.86 \\
PTGS2 & 1.14 & 73.00 & 87.50 & 224.73 \\
RELA & 1.18 & 54.00 & 77.83 & 41.41 \\
SPP1 & 1.25 & 51.00 & 76.50 & 39.41 \\
STAT3 & 1.16 & 72.00 & 86.83 & 132.54 \\
TLR4 & 1.12 & 72.00 & 87.00 & 135.66 \\
\hline
\end{tabular}

3.4. Enrichment Analysis of the Core Targets of Qubi Formula in Treating Psoriasis. In order to further understand the mechanism of "multitarget and multipathway" of Qubi Formula in treating psoriasis, ClueGO plugin was used to perform enrichment analysis of GO-PB and KEGG on core targets and to excavate the biological processes and signaling pathways regulated by Qubi Formula in treating psoriasis. These 27 core targets were involved in several biological process, mainly including nuclear translocation of proteins, cellular response to multiple stimuli (immunoinflammatory factors, oxidative stress, and nutrient substance), lymphocyte activation, regulation of cyclase activity, cell-cell adhesion, and cell death (Figure 4(a)). Moreover, according to the pvalues of enriched pathways and their correlation with psoriasis, we were most interested in the following five representative signal pathways including VEGF, JAK-STAT, TLRs, NF- $\kappa \mathrm{B}$, and lymphocyte differentiation-related pathway (Figure 4(b) and Table 3 ).

\section{Discussion}

Qubi Formula is an experience prescription for psoriasis at the Department of Dermatology in our hospital. It is especially suitable for patients with blood-heat subtype of psoriasis, with radiated skin lesions throughout the whole body, redness, obvious scales, itching and burning, red tongue, and yellow fur. In this formula, SNJ, DH, JYH, and BLG can clear heat, cool blood, and remove toxic materials. MDP and CS are responsible for cooling blood and removing blood stasis. GC is in charge of detoxifying and reconciling medicine. The therapeutic effects of Qubi Formula in the clinical treatment of psoriasis are significant. However, the active ingredients and potential targets of Qubi Formula are unclear, which hinders the further development and application of the prescription.

Network pharmacology is a new strategy for drug design and development based on the rapid development of systematical biology and multidirectional pharmacology. This concept was first proposed by Hopkins AL in 2007, which was switched from previous "disease-single target-single 


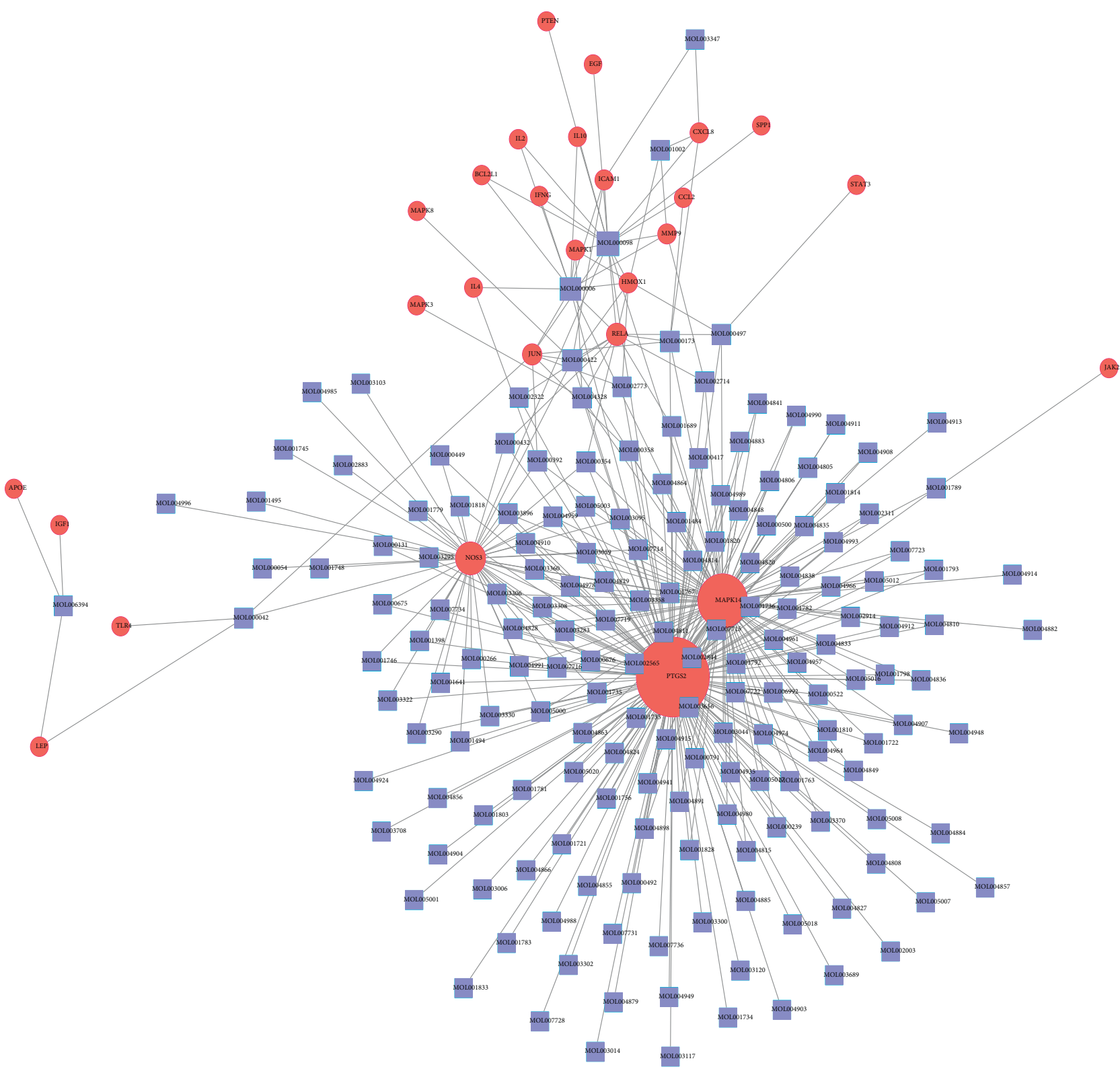

(a)

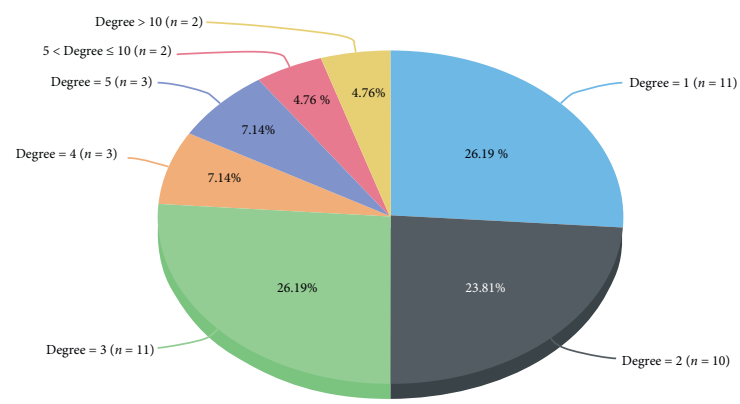

(b)

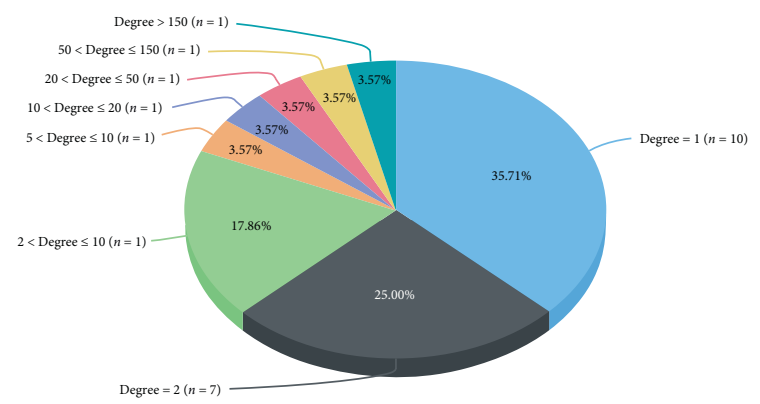

(c)

FIGURE 3: Construction of active ingredients-targets core network for QBF in treating psoriasis (a), and the statistical analysis of the degree of each ingredient (b) and target (c) in the network. All nodes were sorted and calculated according to the degree of freedom, and the node size in the network was associated with degree. 


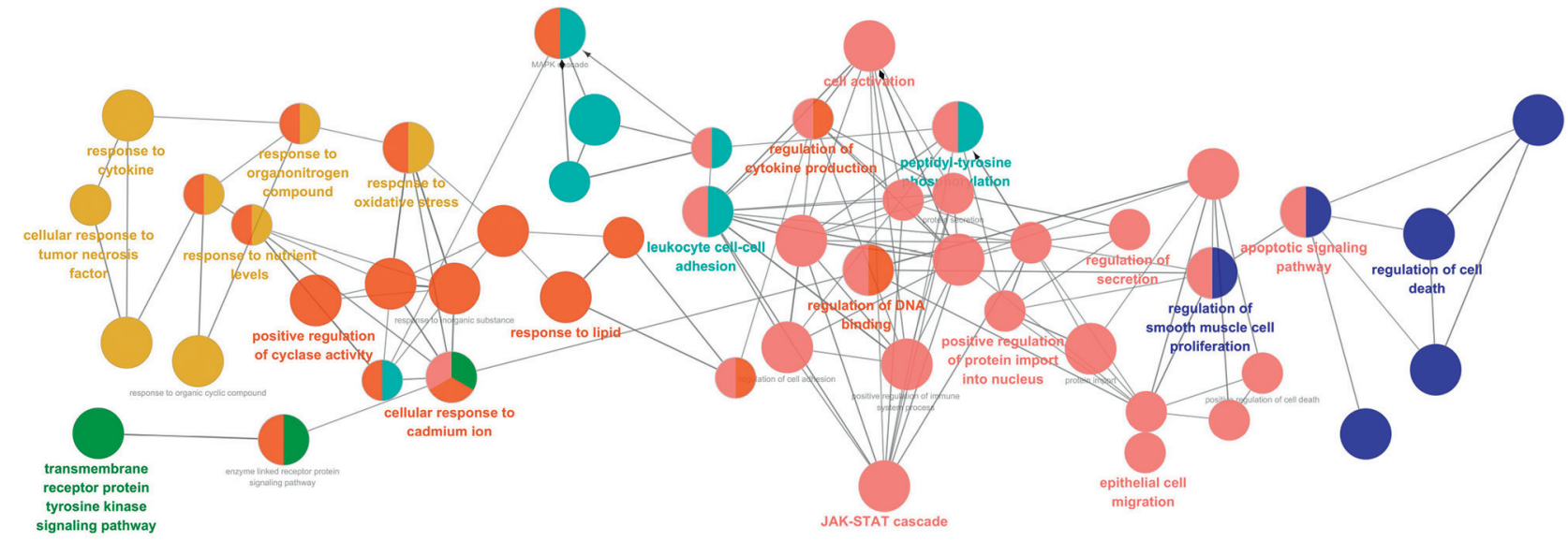

(a)

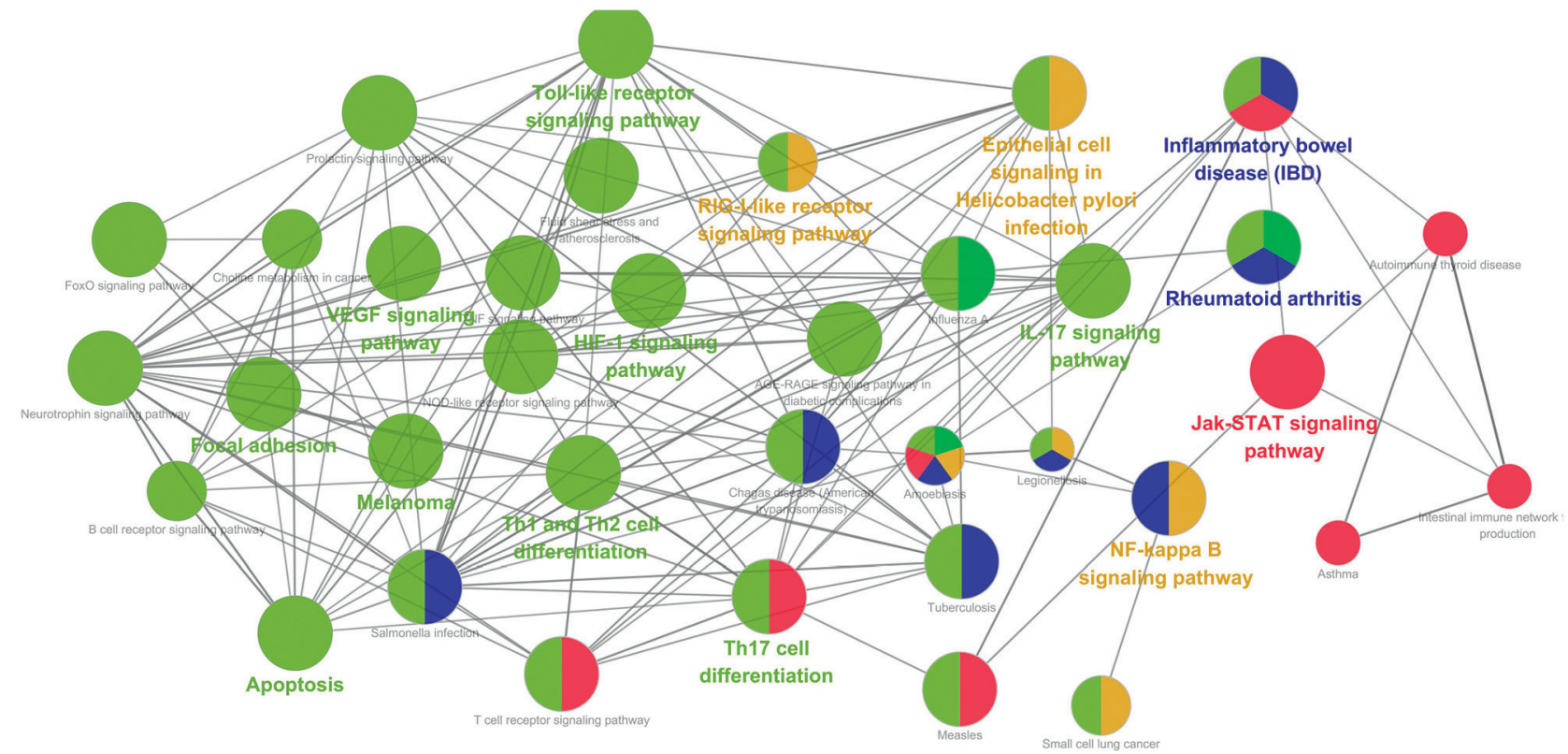

(b)

FIGURE 4: Enrichment analysis of candidate targets for QBF against psoriasis. The enrichment analysis is generated by ClueGO and the most vital term in the group is labeled. Functionally related groups partially overlap. Representative enriched biological process or pathway $(P<0.05)$ interactions among core $\mathrm{QBF}$ targets. The larger circle indicated the greater degree of enrichment, and the closer color suggested the more similar function in biological network. (a) Core QBF targets enriched in the representative biological process. (b) Core QBF targets enriched in the representative signaling pathway.

TABle 3: Representative enriched KEGG pathway of the core targets of Qubi Formula in treating psoriasis.

\begin{tabular}{lcccc}
\hline Pathway & $\begin{array}{c}\text { Gene } \\
\text { count }\end{array}$ & $P$ value & $\begin{array}{c}\text { Pathway } \\
\text { ID }\end{array}$ & Associated genes \\
\hline $\begin{array}{l}\text { Th17 cell differentiation } \\
\text { Th1 and Th2 cell differentiation }\end{array}$ & 11 & $3.87 E-12$ & ko04659 & IFNG, IL2, IL4, JAK2, JUN, MAPK1, MAPK14, MAPK3, MAPK8, \\
RELA, STAT3
\end{tabular}


drug" model of new drug development to the "diseasemultitarget-multidrug" model. This idea coincides with the "holistic view" of TCM. Therefore, the application of the network pharmacology method can provide certain research ideas for discovering the mechanism of Qubi Formula in treating psoriasis.

In this study, a total of 175 potential active ingredients of Qubi Formula in treating psoriasis were screened through a series of network pharmacological methods, which corresponded to 27 core targets. At present, the widely acknowledged histopathological features of psoriasis include four major aspects: the inflammatory infiltration in dermis and epidermis, the abnormal biological behaviours (differentiation, hyperproliferation, and apoptosis) of keratinocytes, metabolic disturbance in skin tissue, and the tortuously increased dermal blood vessels and capillaries [37-41]. Firstly, among the 27 core targets, most of them (PTGS2, ILs, JAK2, STAT3, RELA, CCL2, CXCL8, EGF, IFNG, and TLR4) have been shown to be involved in abnormal inflammatory infiltration, which could regulate the differentiation and chemotaxis of lymphocytes, cytokines produced, and immunological inflammatory reaction in dermis and epidermis [42-48]. Secondly, RELA, MAPKs, JUN, and BCL2L1 are associated with the aberrant biological behaviours of keratinocytes in psoriasis [49-51]. Thirdly, APOE, HMOX1, LEP, IGF1, and SPP1 have been demonstrated to take part in metabolic disturbance (including lipids, peroxides, and carbohydrates) in skin tissue [52-54]. Finally, ICAM1, MM9, and NOS3 are closely associated with endothelial cell proliferation, migration, and adhesion, which are related to the tortuously increased dermal blood vessels and capillaries $[55,56]$.

Enrichment analysis of GO-BP and KEGG on the core targets further suggests that Qubi Formula could intervene in psoriasis through multiple biological processes by acting on several signaling pathways, involving VEGF, JAK-STAT, TLRs, NF- $\kappa \mathrm{B}$, and lymphocyte differentiation-related pathways. As shown in the Figure 4(a), these five signaling pathways cross-talk effects in the network. VEGF signal pathway has been demonstrated to not only induce pathological angiogenesis in psoriatic lesions by regulating the proliferation and differentiation of endothelial cells, but also aggravate the inflammatory response via increasing vascular permeability to promote inflammatory cell infiltration $[57,58]$. Moreover, as it is well known that psoriasis is an inflammatory disease mediated by $\mathrm{T}$ lymphocytes, abnormal differentiation of $\mathrm{T}$ lymphocytes (especially $\mathrm{Th} 1$ and Th17 cells) and excessive secretion of proinflammatory factors (such as ILs) are closely related to the progression of the disease [59]. In this study, we have also demonstrated that there are diverse critical signaling pathways related to $T$ lymphocytes differentiation and proinflammatory factors production (TLRs, JAK-STAT, and NF- $\kappa \mathrm{B}$ ) regulated by QBF on psoriasis therapy [60-62].

Our team has previously confirmed the safety and effectiveness of Qubi Formula in treating psoriasis through clinical observations. Based on the results of this study, we speculate that the regulatory role of Qubi Formula on psoriasis is not unilateral, but is directly or indirectly involved in the comprehensive treatment of the four major pathological factors of psoriasis through multiple signaling pathways associated with immunoinflammatory response, metabolism, and abnormal angiogenesis. Despite the valuable discoveries, there are still certain limitations. In the present study concerning the network pharmacological analysis on Qubi Formula, only the interactions between the components of QBF and the psoriasis-related targets were considered, but the interactions between the ingredients, the dosage of each ingredient, and the effects of the different processing methods of medicinal materials were neglected. Therefore, the obtained results must be verified by further experiments.

\section{Conclusion}

The present study illustrates the systemic "multicompound and multitarget" efficacy of QBF against psoriasis. Moreover, this study also provided a theoretical basis to determine the synergistic effects of TCM in treating diseases and the role of systematic network pharmacology in elucidating the potential mechanisms of action of TCMs. However, as this study was based on data mining and data analysis, further validation studies should be undertaken.

\section{Data Availability}

The data used to support the findings of this study are available from the corresponding author upon request.

\section{Conflicts of Interest}

The authors declare that they have no conflicts of interest regarding the publication of this paper.

\section{Acknowledgments}

The authors thank the members of their laboratory and their collaborators for their research work. This article was funded by the Construction Project of Key Clinical Special Disease of Jiangsu Province Academy of Traditional Chinese Medicine.

\section{Supplementary Materials}

Table S1. Known psoriasis-related targets. Table S2. All the potential targets of QBF. Table S3. QBF shared 104 potential targets with known psoriasis-related targets. Table S4. Topological feature values of all the candidate targets for QBF against psoriasis. (Supplementary Materials)

\section{References}

[1] K. Chen, G. Wang, H. Jin et al., "Clinic characteristics of psoriasis in China: a nationwide survey in over 12000 patients," Oncotarget, vol. 8, no. 28, pp. 46381-46389, 2017.

[2] J. Li, M. Yu, Y. W. Wang et al., "Prevalence of psoriasis and associated risk factors in China: protocol of a nationwide, population-based, cross-sectional study," BMJ Open, vol. 9, no. 7, Article ID e027685, 2019. 
[3] M. Kunz, J. C. Simon, and A. Saalbach, "Psoriasis: obesity and fatty acids," Frontiers in Immunology, vol. 10, p. 1807, 2019.

[4] D. Das, S. Akhtar, S. Kurra, S. Gupta, and A. Sharma, "Emerging role of immune cell network in autoimmune skin disorders: an update on pemphigus, vitiligo and psoriasis," Cytokine \& Growth Factor Reviews, vol. 45, pp. 35-44, 2019.

[5] J. Zhou, D. Sun, L. Xu, L. Sun, S. Fu, and Y. Li, "ADAM33 as a psoriasis susceptibility gene in the Han population of northeastern China," Dermatology, vol. 223, no. 4, pp. 356362, 2011.

[6] X. Yao, S. Hao, and P. Yu, "Association study of the caspase gene family and psoriasis vulgaris susceptibility in northeastern China," BioMed Research International, vol. 2019, Article ID 2417612, 11 pages, 2019.

[7] X. Fan, H. Wang, L. Sun et al., "Fine mapping and subphenotyping implicates ADRA1B gene variants in psoriasis susceptibility in a Chinese population," Epigenomics, vol. 11, no. 4, pp. 455-467, 2019.

[8] Q. Luo, J. Zeng, W. Li et al., "Interaction of MTHFR gene with smoking and alcohol use and haplotype combination susceptibility to psoriasis in Chinese population," Immunologic Research, vol. 66, no. 4, pp. 543-547, 2018.

[9] S. Lorscheid, A. Muller, J. Loffler et al., "Keratinocyte-derived $\mathrm{I} \kappa \mathrm{B} \zeta$ drives psoriasis and associated systemic inflammation," JCI Insight, vol. 422 pages, 2019.

[10] S. Meng, Z. Lin, Y. Wang et al., "Psoriasis therapy by Chinese medicine and modern agents," Chinese Medicine, vol. 13, p. 16, 2018.

[11] J. Deng, D. Yao, C. Lu et al., "Oral Chinese herbal medicine for psoriasis vulgaris: protocol for a randomised, double-blind, double-dummy, multicentre clinical trial," BMJ Open, vol. 7, no. 11, Article ID e014475, 2017.

[12] C.-C. Chiang, W.-J. Cheng, C.-Y. Lin et al., "Kan-Lu-HsiaoTu-Tan, a traditional Chinese medicine formula, inhibits human neutrophil activation and ameliorates imiquimodinduced psoriasis-like skin inflammation," Journal of Ethnopharmacology, vol. 246, p. 112246, 2020.

[13] M. Xu, J. Deng, K. Xu et al., "In-depth serum proteomics reveals biomarkers of psoriasis severity and response to traditional Chinese medicine," Theranostics, vol. 9, no. 9, pp. 2475-2488, 2019.

[14] T.-T. Luo, Y. Lu, S.-K. Yan, X. Xiao, X.-L. Rong, and J. Guo, "Network pharmacology in research of Chinese medicine formula: methodology, application and prospective," Chinese Journal of Integrative Medicine, vol. 26, no. 1, pp. 72-80, 2020.

[15] Z. Liu, F. Guo, Y. Wang et al., "BATMAN-TCM: a bioinformatics analysis tool for molecular mechanism of traditional Chinese medicine," Scientific Reports, vol. 6, p. 21146, 2016.

[16] J. Ru, P. Li, J. Wang et al., "TCMSP: a database of systems pharmacology for drug discovery from herbal medicines," Journal of Cheminformatics, vol. 6, p. 13, 2014.

[17] X. Wang, X. Xu, W. Tao et al., "A systems biology approach to uncovering pharmacological synergy in herbal medicines with applications to cardiovascular disease," Evidence-Based Complementary and Alternative Medicine, vol. 2012, Article ID 519031, 15 pages, 2012.

[18] W. Tao, X. Xu, X. Wang et al., "Network pharmacology-based prediction of the active ingredients and potential targets of Chinese herbal Radix Curcumae formula for application to cardiovascular disease," Journal of Ethnopharmacology, vol. 145, no. 1, pp. 1-10, 2013.

[19] X. Xu, W. Zhang, C. Huang et al., "A novel chemometric method for the prediction of human oral bioavailability,"
International Journal of Molecular Sciences, vol. 13, no. 6, pp. 6964-6982, 2012.

[20] C.-Y. Jia, J.-Y. Li, G.-F. Hao, and G.-F. Yang, "A drug-likeness toolbox facilitates ADMET study in drug discovery," Drug Discovery Today, vol. 25, no. 1, pp. 248-258, 2020.

[21] G. Martin-Ezquerra, M. Sanchez-Regana, and P. UmbertMillet, "Optimization of narrow-band UVB with a $5 \%$ oleic acid cream in the treatment of psoriasis," Journal of Drugs in Dermatology, vol. 6, no. 3, pp. 290-292, 2007.

[22] X.-S. Yao, Y. Ebizuka, H. Noguchi et al., "Biologically active constituents of Arnebia euchroma: structure of arnebinol, an ansa-type monoterpenylbenzenoid with inhibitory activity on prostaglandin biosynthesis," Chemical \& Pharmaceutical Bulletin, vol. 39, no. 11, pp. 2956-2961, 1991.

[23] P. Li, J. Chen, J. Wang et al., "Systems pharmacology strategies for drug discovery and combination with applications to cardiovascular diseases," Journal of Ethnopharmacology, vol. 151, no. 1, pp. 93-107, 2014.

[24] Y. Wang, S. Zhang, F. Li et al., "Therapeutic target database 2020: enriched resource for facilitating research and early development of targeted therapeutics," Nucleic Acids Research, vol. 48, no. 1, pp. D1031-D1041, 2019.

[25] D. S. Wishart, C. Knox, A. C. Guo et al., "DrugBank: a knowledgebase for drugs, drug actions and drug targets," Nucleic Acids Research, vol. 36, pp. D901-D906, 2018.

[26] N. Rappaport, M. Twik, I. Plaschkes et al., "MalaCards: an amalgamated human disease compendium with diverse clinical and genetic annotation and structured search," Nucleic Acids Research, vol. 45, no. 1, pp. D877-D887, 2017.

[27] J. Pinero, N. Queralt-Rosinach, A. Bravo et al., "DisGeNET: a discovery platform for the dynamical exploration of human diseases and their genes," Database, vol. 2015, Article ID bav028, 2015.

[28] The UniProt Consortium, "UniProt: the universal protein knowledgebase," Nucleic Acids Research, vol. 45, no. 1, pp. D158-D169, 2017.

[29] C. V. Mering, M. Huynen, D. Jaeggi et al., "STRING: a database of predicted functional associations between proteins," Nucleic Acids Research, vol. 31, no. 1, pp. 258-261, 2003.

[30] C.-H. Chin, S.-H. Chen, H.-H. Wu, C.-W. Ho, M.-T. Ko, and C.-Y. Lin, "cytoHubba: identifying hub objects and subnetworks from complex interactome," BMC Systems Biology, vol. 8, no. 4, p. S11, 2014.

[31] G. Bindea, B. Mlecnik, H. Hackl et al., "ClueGO: a Cytoscape plug-in to decipher functionally grouped gene ontology and pathway annotation networks," Bioinformatics, vol. 25, no. 8, pp. 1091-1093, 2009.

[32] H. Chen, C. Lu, H. Liu et al., "Quercetin ameliorates imiquimod-induced psoriasis-like skin inflammation in mice via the NF- $\kappa \mathrm{B}$ pathway," International Immunopharmacology, vol. 48, pp. 110-117, 2017.

[33] J. Lv, D. Zhou, Y. Wang et al., "Effects of luteolin on treatment of psoriasis by repressing HSP90," International Immunopharmacology, vol. 79, p. 106070, 2020.

[34] C. Liu, H. Liu, C. Lu et al., "Kaempferol attenuates imiquimodinduced psoriatic skin inflammation in a mouse model," Clinical \& Experimental Immunology, vol. 198, no. 3, pp. 403-415, 2019.

[35] X. Duan, Y. Cheng, L. Gao, L. Li, T. Wang, and M. Zhang, "Evaluation of the potential association between NOS gene polymorphisms (iNOS G-954C and eNOS G894T) and psoriasis," Annals of Dermatology, vol. 28, no. 1, pp. 110-112, 2016.

[36] B. Yalçin, G. G. Tezel, N. Arda, M. Erman, and N. Alli, "Vascular endothelial growth factor, vascular endothelial 
growth factor receptor-3 and cyclooxygenase-2 expression in psoriasis," Analytical and Quantitative Cytology and Histology, vol. 29, no. 6, pp. 358-364, 2007.

[37] L. Capriotti, B. Didona, S. Madonna et al., "Eosin treatment for psoriasis reduces skin leukocyte infiltration and secretion of inflammatory chemokines and angiogenic factors," European Journal of Dermatology, vol. 28, no. 4, pp. 457-466, 2018.

[38] N. Jadhav, N. Nadkarni, and S. Patil, "A study on the association of psoriasis with metabolic disorders," Journal of the Association of Physicians of India, vol. 67, no. 4, pp. 52-54, 2019.

[39] J. M. Fernandez-Armenteros, X. Gomez-Arbones, M. ButiSoler et al., "Psoriasis, metabolic syndrome and cardiovascular risk factors. A population-based study," Journal of the European Academy of Dermatology and Venereologyl, vol. 33, no. 1, pp. 128-135, 2019.

[40] N. Garzorz-Stark and K. Eyerich, "Psoriasis pathogenesis: keratinocytes are back in the spotlight," Journal of Investigative Dermatology, vol. 139, no. 5, pp. 995-996, 2019.

[41] F. Benhadou, D. Mintoff, and V. del Marmol, "Psoriasis: keratinocytes or immune cells-which is the trigger?" Dermatology, vol. 235, no. 2, pp. 91-100, 2019.

[42] G. Shi, T. Wang, S. Li et al., "TLR2 and TLR4 polymorphisms in southern Chinese psoriasis vulgaris patients," Journal of Dermatological Science, vol. 83, no. 2, pp. 145-147, 2016.

[43] L. Hsu and A. W. Armstrong, "JAK inhibitors: treatment efficacy and safety profile in patients with psoriasis," Journal of Immunology Research, vol. 2014, Article ID 283617, 7 pages, 2014.

[44] W. Sun, Y. Gao, X. Yu et al., ““Psoriasis 1” reduces psoriasislike skin inflammation by inhibiting the VDRmediated nuclear NFkappaB and STAT signaling pathways," Molecular Medicine Reports, vol. 18, no. 3, pp. 2733-2743, 2018.

[45] C. Schuster, A. Huard, E. Sirait-Fischer et al., "S1PR4-dependent CCL2 production promotes macrophage recruitment in a murine psoriasis model," European Journal of Immunology, vol. 50, no. 6, 2020.

[46] M. Szterling-Jaworowska, A. Baran, H. Myśliwiec, and I. Flisiak, "Effect of psoriasis activity and topical treatment on plasma epidermal growth factor (EGF) and its soluble receptor (sEGFR)," Journal of Dermatological Treatment, vol. 29, no. 2, pp. 135-139, 2018.

[47] J. L. Harden, L. M. Johnson-Huang, M. F. Chamian et al., "Humanized anti-IFN- $\gamma$ (HuZAF) in the treatment of psoriasis," Journal of Allergy and Clinical Immunology, vol. 135, no. 2, pp. 553-556, 2015.

[48] Y. K. Kim, C. W. Pyo, H. B. Choi et al., "Associations of IL-2 and IL-4 gene polymorphisms with psoriasis in the Korean population," Journal of Dermatological Science, vol. 48, no. 2, pp. 133-139, 2015.

[49] Y. Xiong, H. Chen, L. Liu et al., "microRNA-130a promotes human keratinocyte viability and migration and inhibits apoptosis through direct regulation of STK40-mediated NF$\kappa \mathrm{B}$ pathway and indirect regulation of SOX9-meditated JNK/ MAPK pathway: a potential role in psoriasis," DNA and Cell Biology, vol. 36, no. 3, pp. 219-226, 2017.

[50] A. G. Abdou, A. H. Marae, M. Shoeib, G. Dawood, and E. Abouelfath, "C-Jun expression in lichen planus, psoriasis, and cutaneous squamous cell carcinoma, an immunohistochemical study," Journal of Immunoassay and Immunochemistry, vol. 39, no. 1, pp. 58-69, 2018.

[51] T. Yamamoto and K. Nishioka, "Alteration of the expression of Bcl-2, Bcl-x, Bax, Fas, and Fas ligand in the involved skin of psoriasis vulgaris following topical anthralin therapy," Skin Pharmacology and Physiology, vol. 16, no. 1, pp. 50-58, 2003.

[52] Y. Han, T. Liu, and L. Lu, "Apolipoprotein E gene polymorphism in psoriasis: a meta-analysis," Archives of Medical Research, vol. 44, no. 1, pp. 46-53, 2013.

[53] C. Hanselmann, C. Mauch, and S. Werner, "Haem oxygenase1: a novel player in cutaneous wound repair and psoriasis?" Biochemical Journal, vol. 353, no. 3, pp. 459-466, 2001.

[54] Y. Kong, S. Zhang, R. Wu et al., "New insights into different adipokines in linking the pathophysiology of obesity and psoriasis," Lipids in Health and Disease, vol. 18, no. 1, p. 171, 2019.

[55] A. L. Bressan, B. L. S. Picciani, L. Azulay-Abulafia et al., "Evaluation of ICAM-1 expression and vascular changes in the skin of patients with plaque, pustular, and erythrodermic psoriasis," International Journal of Dermatology, vol. 57, no. 2, pp. 209-216, 2018.

[56] P. Coto-Segura, E. Coto, A. Mas-Vidal et al., "Influence of endothelial nitric oxide synthase polymorphisms in psoriasis risk," Archives of Dermatological Research, vol. 303, no. 6, pp. 445-449, 2018.

[57] M. E. Marina, I. I. Roman, A.-M. Constantin, C. M. Mihu, and A. D. Tătaru, "VEGF involvement in psoriasis," Medicine and Pharmacy Reports, vol. 88, no. 3, pp. 247-252, 2015.

[58] N. Malecic and H. S. Young, "Novel investigational vascular endothelial growth factor (VEGF) receptor antagonists for psoriasis," Expert Opinion on Investigational Drugs, vol. 25, no. 4, pp. 455-462, 2016.

[59] S. C. Furiati, J. S. Catarino, M. V. Silva et al., "Th1, Th17, and treg responses are differently modulated by TNF-alpha inhibitors and methotrexate in psoriasis patients," Scientific Reports, vol. 9, no. 1, p. 7526, 2019.

[60] F. Zhou, Z. Zhu, J. Gao et al., "NFKB1 mediates Th1/Th17 activation in the pathogenesis of psoriasis," Cellular Immunology, vol. 331, pp. 16-21, 2018.

[61] A. Adamson, K. Ghoreschi, M. Rittler et al., "Tissue inhibitor of metalloproteinase 1 is preferentially expressed in Th1 and Th17 T-helper cell subsets and is a direct STAT target gene," PLoS One, vol. 8, no. 3, Article ID e59367, 2013.

[62] W. Jiang, F.-G. Zhu, L. Bhagat et al., "A Toll-like receptor 7, 8, and 9 antagonist inhibits Th1 and Th17 responses and inflammasome activation in a model of IL-23-induced psoriasis," Journal of Investigative Dermatology, vol. 133, no. 7, pp. 1777-1784, 2013. 